\title{
Preferred practice patterns of ophthalmologists in management of viral conjunctivitis
}

\author{
Tania Moudgil ${ }^{1}$, Barinder Kaur ${ }^{2 *}$, Gaganpreet Singh $^{3}$
}

${ }^{\mathbf{1}}$ Associate Professor, ${ }^{2}$ Assistant Professor, ${ }^{3}$ Junior Resident, Dept. of Ophthalmology, Punjab Institute of Medical Sciences, Jalandhar, Punjab, India

\section{Article Info}

Received: $28^{\text {th }}$ February, 2019

Accepted: $15^{\text {th }}$ April, 2019

Published Online: $9^{\text {th }}$ September, 2019

Keywords: Antiviral agents, Conjunctivitis, Cross sectional studies, Ophthalmologists, Viral.

\begin{abstract}
Introduction: Different ophthalmologists follow different protocols for diagnosing and treating the viral conjunctivitis. We conducted this study to know the current trends of knowledge, attitude and practices in management of viral conjunctivitis.

Aims: 1: To determine the knowledge, attitude and practices of ophthalmologist in diagnosing viral conjunctivitis; 2: To determine the knowledge, attitude and practices of ophthalmologist in treatment of viral conjunctivitis.

Design: A Prospective descriptive cross sectional study based on questionnaire survey will be done amongst ophthalmologists of Punjab, Gujarat, Haryana and Maharashtra.

Materials and Methods: A prevalidated questionnaire was distributed manually and by email to ophthalmologists in different parts of India. The questionnaire covered their knowledge about diagnosis and treatment of viral conjunctivitis. It also covered their preferred practice pattern for managing a case of viral conjunctivitis. The data was collected and analysed on SPSS 24 software. Thematic analysis of qualitative data obtained was done. Results: 205 responses were received from ophthalmologists across various parts of India. The basis of diagnosis for a case of viral conjunctivitis was clinical by $88.5 \%$ of ophthalmologists. Signs looked by ophthalmologists were mainly congestion $(80 \%)$, discharge $(72.19 \%)$ and follicles $(48.3 \%) .92 \%$ responded that no investigations are required for forming diagnosis. An overall criterion used by ophthalmologists was $85 \%$ clinical picture along with history, $12 \%$ use diagnosis of exclusion and $3 \%$ used certain investigations for diagnosis. Ophthalmologists use the treatment plan as lubricants $(86.3 \%)$, Antibacterial (66.7\%), steroids (35.3\%), antiviral (15.7\%), oral vitamin C (25.5\%) and other $(11.8 \%)$. For prevention of viral conjunctivitis precautions used for self were hand hygiene (washing hand [98\%] and sanitizer like sterllium [25\%]), use of gloves (2\%), clean slit lamp, chair and other instruments: with sanitizer $(82.1 \%) .98 \%$ ophthalmologists responded that they educate the patients regarding precautions to be followed for treatment of viral conjunctivitis. Among precautions following themes emerged: dark goggles (29.2\%) and hygiene $(98.9 \%)$.

Conclusions: Viral conjunctivitis is diagnosed mainly on clinical picture. Treatment is mainly supportive consisting of lubricants, oral vitamin $\mathrm{C}$ and topical antibiotics. Topical steroids can be a useful in cases of keratitis and pseudomembrane associated with viral conjunctivitis. Topical antivirals are given only in cases of recurrent keratitis secondary to viral conjunctivitis.
\end{abstract}

\section{Introduction}

Viral conjunctivitis is the most common type of infectious conjunctivitis and is more prevalent in hot humid seasons. ${ }^{1,2}$ It is very contagious. Patients are in lot of discomfort as its symptoms stay for a longer time and it does not respond to conventional treatment. It may have sequelae if corneal involvement is there and patients discomfort and blurring of vision will stay for a longer time. A significant number of days of work are lost by patients due to viral conjunctivitis. Some studies have even reported depression due to chronic nature of the disease. ${ }^{3}$
It is important to differentiate viral conjunctivitis from other sight-threatening eye diseases and other causes of conjunctivitis that have similar clinical presentation. ${ }^{4}$ However it is often difficult to diagnose based on clinical appearance and in the early stages. It is often associated with a red eye or superficial keratitis common to herpes and other infections. Further to make appropriate decisions about further testing and treatment differs for different ophthalmologists. Even they follow different management protocol, yet no established treatment exists. ${ }^{5}$

\footnotetext{
*Corresponding Author: Barinder Kaur, Assistant Professor, Dept. of Ophthalmology, Punjab Institute of Medical Sciences, Jalandhar, Punjab, India

Email: taniamoudgil1981@gmail.com

http://doi.org/10.18231/j.ijceo.2019.094
} 
Hence we propose this study to know the preferred practice pattern of ophthalmologists for management of viral conjunctivitis.

\section{Materials and Methods}

A prevalidated questionnaire (Annexure 1) was distributed manually and by email to ophthalmologists in different parts of India. For validation of the questionnaire the content was validated by the ophthalmologists of the institute and external validation was done by ophthalomologists from outside the institute. I-CVI was calculated and questions with I-CVI less than 0.80 were excluded. The questionnaire covered their knowledge about diagnosis and treatment of viral conjunctivitis. It also covered their preferred practice pattern for managing a case of viral conjunctivitis. Details of the questionnaire: years of practice, prevalence of viral conjunctivitis in their OPD, basis of their diagnosis for viral conjunctivitis, precautions they follow and whether they are educating the patients for precautions to follow to prevent the spread of the disease, line of management, use of steroids, if steroids used the time of starting the steroids, the frequency and preferable steroid. The data was collected and analysed on SPSS 24 software. Thematic analysis of qualitative data obtained was done.

\section{Results}

Demographic profile: Area of practice: $67.3 \%$ of ophthalmologists practiced in urban, $21.2 \%$ in semi Urban and $11.5 \%$ in rural. $51.9 \%$ practices more than 10 years, $28.8 \%$ practiced between $5-10$ years and $19.2 \%$ practiced for last 5 years. The most common aetiology of conjunctivitis they see in their practice was $47.1 \%$ as viral, $41.2 \%$ as allergic, $9.8 \%$ as bacterial and $1.8 \%$ reported other causes. $62.7 \%$ reported that they see less than 5 cases of viral conjunctivitis in a week, $35.3 \%$ reported that they see 5-10 cases per week and $2 \%$ reported that they see more than 10 cases per week.

\section{Diagnosis}

The basis of diagnosis for a case of viral conjunctivitis was clinical by $88.5 \%$ of ophthalmologists, $7.7 \%$ made diagnosis of exclusion and $3.8 \%$ reported other methods for forming diagnosis.

\section{Symptoms}

Among symptoms reported by ophthalmologists pointing towards clinical diagnosis mainly were foreign body sensation, sudden onset with rapid progress and epidemic. Other symptoms: epidemic, history of contact, hot humid season, watering and association with upper respiratory tract infection

Signs looked by ophthalmologists were mainly congestion $(80 \%)$, discharge $(72.19 \%)$ and follicles $(48.3 \%)$. Amongst other signs were papillae, petechial haemorrhages, superficial punctuate keratitis, lymphadenopathy, unilateral, membrane and not responding to antibiotics. (Fig. 1)

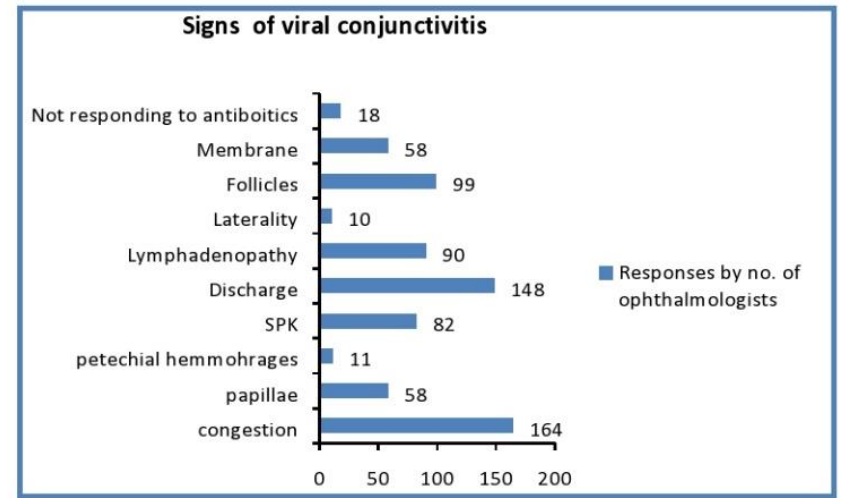

Fig. 1: Signs of viral conjunctivitis as reported by ophthalmologists across various parts of India

\section{Investigations}

In response to investigation requirement: $92 \%$ responded that no investigations are required for forming diagnosis.3.8\% responded that they did investigations in some doubtful cases in form of conjunctival swab. $2.2 \%$ looked for corneal sensations. $2 \%$ were not sure. Among investigations: gram staining, Nested polymerase chain reaction (nPCR) and restriction fragment length polymorphism (RFLP) technique were reported.

An overall criteria used by ophthalmologists was $85 \%$ used clinical picture along with history, $12 \%$ use diagnosis of exclusion and 3\% used certain investigations for diagnosis.

\section{Treatment Plan}

Ophthalmologists use the treatment plan as lubricants (86.3\%), Antibacterials (66.7\%), steroids (35.3\%), antivirals (15.7\%), oral vitamin C (25.5\%) and other (11.8\%).

\section{Use of Steroids}

$36.5 \%$ ophthalmologists use steroids at some point of the treatment of viral conjunctivitis, $32.7 \%$ chose the option of maybe for use of steroids and $30.8 \%$ never give steroids in a treatment plan for viral conjunctivitis. The choice for steroids was Loteprednol (29.2\%), Flurometholone (29.2\%), Prednisolone $(4.2 \%)$, Dexamethasone $(6.3 \%)$, any other steroid preparation $(4.2 \%)$ and do not prescribe steroids $(27.1 \%)$.

\section{Rationale behind avoidance of Steroids}

Among reasons the most common responses were aggravated secondary infection and in case of purulent discharge and increased duration of shedding of adenovirus.

\section{Rationale behind use of Steroids}

To decrease inflammation (70\%), papillary response $(20 \%)$, subeptithelial lesions (45\%), corneal infiltrates $(2 \%)$, shorten recovery time (1.8\%), pseudomambranous conjunctivitis $(35.2 \%)$ and in case of patient's need for urgent recovery $(0.1 \%)$.

\section{Time to start steroid therapy}

At the time of diagnosis (20.4\%), add it after initial therapy if symptoms are not relieved (59.2\%) and $20.4 \%$ do not give it at all. 


\section{Frequency of Steroid Drops}

$61.2 \%$. Use steroids less than 4times/ day, $14.3 \%$ use it $4-6$ times/ day, $4.1 \%$ use it $>6$ times/ day and $20.4 \%$ do not prescribe at all.

\section{Use of Antiviral (topical or oral)}

$15.7 \%$ reported use of topical antivirals in case of recurrent viral keratoconjunctivitis. Rest $84.3 \%$ did not prescribed antivirals at any point of treatment. The choice of topical antiviral for cases of recurrent viral keratoconjunctivitis was gancyclovir $0.15 \%$ three times a day.

Rationale behind avoiding topical or oral antivirals: Risks outweigh benefits, topical deposition of antivirals.

Rationale behind usage by some ophthalmologists: Recurrent cases do not respond to other forms of treatment. It decreases the viral shedding and shortens the treatment.

\section{Prevention of Viral Conjunctivitis}

For Self: hand hygiene (washing hand [98\%] and sanitizer like sterllium [25\%]), use of gloves (2\%), clean slit lamp, chair and other instruments: with sanitizer $(82.1 \%)$, Chin paper strips $(15 \%)$, Cleaning probable places that patient touched with isopropyl alcohol swabs (1\%), Avoiding refraction or other procedures, no non contact tonometry or AR(10\%), Knowledge to patients and staff, avoid touching eye

Education to Patients: 98\% ophthalmologists responded that they educate the patients regarding precautions to be followed for treatment of viral conjunctivitis. Among precautions following themes emerged: dark goggles $(29.2 \%)$, hygiene $(98.9 \%)$ : use hand sanitizer or wash their hands thoroughly after touching their eyes or nasal secretions, use disposable tissues to wipe eyes and discard immediately, avoid touching the noninfected eye after touching the infected eye (22.7\%), avoid sharing towels or pillows, linens and cosmetics (15.9\%), relative isolation (15\%): avoid swimming in pools. Stay at home from school or work, contact lens users should be instructed to discontinue contact lens wear until signs and symptoms resolved (2\%), special instructions regarding steroids if they are used $(2 \%)$.

\section{Discussion}

Viral conjunctivitis is the most common ${ }^{1,6}$ type of acute conjunctivitis though it is many a times misdiagnosed as bacterial conjunctivitis and the treatment for same has also not been established. This study focussed on the preferred practice patterns of ophthalmologists from various parts of India for management of viral conjunctivitis.

In this questionnaire based survey 205 ophthalmologists responded and preferred practice patterns for management of viral conjunctivitis were established. In our study, viral conjunctivitis was reported as the most common cause of acute conjunctivitis. This can be attributed to the fact that study was done in months of June- July. Hot humid climate has been reported as one of the predisposing factors for epidemics of viral conjunctivitis. Many studies have reported similarly, viral conjunctivitis as the most common cause of conjunctivitis. However we could not find studies for same in India. Some studies have reported viral as a cause up to $80 \%$ of all cases of acute conjunctivitis.

Diagnosis of viral conjunctivitis as preferred by clinicians according to our study was based on clinical picture rather than laboratory investigations. However in one study it was stated that the rate of clinical accuracy in diagnosing viral conjunctivitis is less than $50 \%$ compared with laboratory confirmation. ${ }^{7,8}$ many cases are misdiagnosed as bacterial conjunctivitis. Yet, in our study the gold standard was clinical diagnosis.

In symptoms, which ophthalmologists reported for forming a provisional diagnosis were foreign body sensation, sudden onset with rapid progress, epidemic, hot humid season, watering and associated with upper respiratory tract infection. The diagnosis is confirmed by signs of superficial congestion, mucoid or watery discharge, follicles, papillae, petechial haemorrhages, superficial punctuates keratitis, lymphadenopathy, unilateral and membrane or pseudomembrane. Some ophthalmologists reported that they formed diagnosis of exclusion or if the patient doesn't respond to antibiotics. This is in correspondence to the findings reported in other studies for features of adenoviral conjunctivitis which amount to $65 \%$ and $90 \%$ of cases of viral conjunctivitis. ${ }^{8-10}$ Symptoms and signs associated have been reported as pharyngoconjunctival fever characterized by abrupt onset of high fever, pharyngitis, and bilateral conjunctivitis, and by periauricular lymph node enlargement, whereas epidemic keratoconjunctivitis is more severe and presents with watery discharge, hyperemia, chemosis, and ipsilateral lymphadenopathy. ${ }^{10}$

As mentioned earlier, most of the ophthalmologists in our study did not depend on any investigations for diagnosis of viral conjunctivitis mainly because they depended on the clinical picture and also most of them did not have facilities for any investigations. Among investigations if any done were conjunctival swab for gram positive or negative staining. However, according to Sambursky $\mathrm{R}$ et $\mathrm{al}^{11}$ a rapid antigen test should be used to diagnose viral conjunctivitis and avoid inappropriate use of antibiotics.

Treatment prescribed by ophthalmologists in our study was mainly supportive. Clinical management for viral conjunctivitis as per other studies was also targeted to provide relief of symptoms, as well as shorten the duration of infection. ${ }^{12}$ In our study treatment plan consisted of topical antibiotics, lubricants and oral vitamin C. Lubricants and Vitamin $\mathrm{C}$ have been reported as part of supportive treatment for viral conjunctivitis by many other studies. ${ }^{13}$ But use of topical antibiotics as a part of treatment regimen has not been supported by some studies denoting that it does not protect against secondary infections, and their use may complicate the clinical presentation by causing allergy and toxicity, leading to delay in diagnosis of other possible ocular diseases. ${ }^{14}$

On use of steroids, only $20.4 \%$ of ophthalmologists never prescribe topical steroids but rest of the ophthalmologists recommended steroids for viral conjunctivitis if the patient developed keratitis or 
pseudomembrane. The rationale mentioned for recommending steroids is that it is a potent antiinflammatory agent. The ophthalmologists who did not prescribe steroids cited reasons as increased duration of adenovirus shedding and aggravated secondary infection associated with use of topical steroids. These indications for using steroids and caution behind use of steroids in cases of viral conjunctivitis are in concordance to a study reported by AM Phios. ${ }^{15}$ It was reported that one should carefully consider the risks involved with the use of a corticosteroid for symptomatic relief of EKC and its potential to prolong an infection. However ophthalmologists in our study reported otherwise. Use of topical antiviral drugs as part of definitive treatment was not supported by the ophthalmologists in our study. Among reasons cited for not using antivirals was that the strain of viral conjunctivitis keeps on changing and an antiviral specific for certain strain may not be effective for other strain. Also the risk benefit ratio was cited as questionable. But, Tabbara K.F. et al ${ }^{16}$ reported that topical ganciclovir $0.15 \%$ ophthalmic ointment is safe and effective for the treatment of adenoviral conjunctivitis. But he also added that the effects of ganciclovir on adenoviral keratoconjunctivitis need to be demonstrated on a larger scale as well as show its effectivity against adenoviral strains and also in other countries.

Among other treatment plans mentioned in literature the efficacy of topical dexamethasone $0.1 \%$ /povidoneiodine $0.4 \%$ (FST-100) in reducing clinical symptoms and infectious viral titres has been demonstrated to have rapid improvement of conjunctival injection and discharge with a decrease in viral titres at the same time..$^{17,18}$

Viral conjunctivitis secondary to adenoviruses is highly contagious, and the risk of transmission has been estimated to be $10 \%$ to $50 \% .4,16,19$ The virus spreads through direct contact via contaminated fingers, medical instruments, swimming pool water, or personal items. In one of the studies, it has been reported that $46 \%$ of infected people had positive cultures grown from swabs of their hands. ${ }^{16}$ Because of the high rates of transmission, hand washing, strict instrument disinfection, and isolation of the infected patients from the rest of the clinic has been advocated..$^{20-22} \mathrm{In}$ our study, precautions taken by ophthalmologists for prevention of viral conjunctivitis to self and hospital personnel were avoiding touching of eye, hand hygiene, use of gloves, cleaning of equipment used using appropriate disinfectants like isopropyl alcohol and avoiding any invasive or contact and educating staff members. Lakkis et al. ${ }^{23}$ also recommend cleaning all large surfaces in an optometric practice with isopropyl alcohol tissues, 30\% alcohol solution or sodium hypochlorite solution (obtained with a $1: 5$ dilution of $5 \%$ household bleach) $.98 \%$ of the ophthalmologists educated the patients regarding the disease, its prevention and precaution regarding the treatment. For prevention hygiene and fomite borne nature of the disease was shared by ophthalmologists as part of education to the patient and regarding treatment, special instructions regarding steroids was also given.

\section{Conclusion}

Viral conjunctivitis is most common type of acute conjunctivitis seen by ophthalmologists in hot and humid season. The diagnosis is formulated mainly on basis of clinical picture which includes mostly unilateral involvement along with foreign body sensation and sudden onset with rapid progress. Among clinical features which are pathognomic of viral conjunctivitis are superficial congestion, discharge, follicles, papillae, petechial hemmorhages, superficial punctuate keratitis, lymphadenopathy and pseudomembrane. Treatment recommended is supportive lubricants, oral vitamin $\mathrm{C}$ and topical antibiotics. Topical antivirals are of limited use. Among them topical Gancyclovir $0.15 \%$ three times a day can be given in case of recurrent keratitis secondary to viral conjunctivitis.

\section{Source of Funding: None.}

\section{Conflict of Interest: None.}

\section{References}

1. Azari AA, Barney NP. Conjunctivitis: a systematic review of diagnosis and treatment. JAMA. 2013;310(16):1721-9.

2. Ford E, Nelson KE, Warren D. Epidemiology of epidemic keratoconjunctivitis. Epidemiol Rev. 1987;9:244-61.

3. Gupta AK, Sarin GS. Serum complement component depression during acute adenovirus conjunctivitis. $\mathrm{Br} \mathrm{J}$ Ophthalmol. 1984;68(5):350-2.

4. Kaufman HE. Adenovirus advances: new diagnostic and therapeutic options. Curr Opin Ophthalmol. 2011;22(4):290-3.

5. Skevaki CL, Galani IE, Pararas MV, Giannopoulou KP, Tsakris A. Treatment of viral conjunctivitis with antiviral drugs. Drugs. 2011;12;71(3):331-47.

6. Epling J. Bacterial conjunctivitis. BMJ Clin Evid. 2010;2010:0704.

7. O'Brien TP, Jeng BH, McDonald M, Raizman MB. Acute conjunctivitis: truth and misconceptions. Curr Med Res Opin. 2009;25(8):1953-61.

8. Madhavan HN. Laboratory investigations on viral and Chlamydia trachomatis infections of the eye: Sankara Nethralaya experiences. Indian J Ophthalmol. 1999;47(4):2416.

9. Weber CM, Eichenbaum JW. Acute red eye. Differentiating viral conjunctivitis from other, less common causes. Postgrad Med. 1997;101(5):185-6.

10. Bialasiewicz A. Adenoviral keratoconjunctivitis. Sultan Qaboos Univ Med J. 2007;7(1):15-23.

11. Sambursky R, Tauber S, Schirra F, Kozich K, Davidson R, Cohen EJ. The RPS adeno detector for diagnosing adenoviral conjunctivitis. Ophthalmol. 2006;113(10):1758-64.

12. Chigbu DI, Labib BA. Pathogenesis and management of adenoviral keratoconjunctivitis. Infect Drug Resist. 2018;11:981-93.

13. American Academy of Ophthalmology. Cornea/External Disease Panel. Preferred Practice Pattern Guidelines: Conjunctivitis-Limited Revision. American Academy of Ophthalmology; San Francisco, CA: 2011.

14. Mahmood AR, Narang AT. Diagnosis and management of the acute red eye. Emerg Med Clin North Am. 2008;26(1):35-55.

15. AM Phios. Epidemic keratoconjunctivitis: A review of current concepts and management. J Optom. 2013;6(2):69-74. 
18. Tabbara K.F., Jarade E.F. Ganciclovir effects in adenoviral keratoconjunctivitis [abstract 3111-

B253] ARVO. 2001;42:S579.

19. Pelletier J.S., Stewart K., Trattler W. A combination povidoneiodine $0.4 \%$ /dexamethasone $0.1 \%$ ophthalmic suspension in the treatment of adenoviral conjunctivitis. $A d v$ Ther. 2009;26:776-83.

20. Clement C., Capriotti J.A., Kumar M. Clinical and antiviral efficacy of an ophthalmic formulation of dexamethosone povidone-iodine in a rabbit model of adenoviral keratoconjunctivitis. Invest Ophthalmol Vis Sci. 2011;52:33944.

21. Høvding G. Acute bacterial conjunctivitis. Acta Ophthalmol. 2008;86(1):5-17.

22. Azar MJ, Dhaliwal DK, Bower KS. Possible consequences of shaking hands with your patients with epidemic keratoconjunctivitis. Am J Ophthalmol. 1996;121(6):711-2.

23. Warren D, Nelson KE, Farrar JA. A large outbreak of epidemic keratoconjunctivitis: problems in controlling nosocomial spread. J Infect Dis. 1989;160(6):938-43.

24. Hamada N, Gotoh K, Hara K. Nosocomial outbreak of epidemic kerato conjunctivitis accompanying environmental contamination with adenoviruses. J Hosp Infect. 2008;68(3):262-8.

25. Lakkis C., Lian K.Y., Napper G. Infection control guidelines for optometrists 2007. Clin Exp Optom. 2007;6:434-44.

How to cite this article: Moudgil T, Kaur B, Singh G. Preferred practice patterns of ophthalmologists in management of viral conjunctivitis. Indian J Clin Exp Ophthalmol 2019;5(3):390-4. 